TÀI LIỆ THAM KHẢO

1. Nguyễ̃n Thị Ngọc Ánh, Nguyễn Thị Kim Liên, Nguyển Thị Thu Hà (2018). Tác dụng giảm đau và cải thiện tầm vận động cột sống cổ của điện châm kết hợp bài tập cột sống cổ trên bệnh nhẩn đau vai gáy do thoái hoá cột sống cổ. Tạp chí Y học Việt Nam, 87-91.

2. Bồ mốn phục hôi chức năng - Trường Đại hoc Y Hà Nô̂i (2017). Phục hồi chức năng (Dùng cho bác sĩ định hướng chuyên khoa). Nhà xuất bản Y hoc, 42-45.

3. Bộ y tế (2008), Quy trình kỹ thuật Y học cổ truyển, Quy trình 46 (Điện châm điều trị hội chứng vai gáy).
4. Mai Trung Dũng (2014). Đánh giá kết quả điều tri kết hợp tâp con lăn Doctor100 trên bệnh nhân hối chứng cổ vai cánh tay do thoái hóa cột sống cổ. Luận văn Bác sĩ chuyên khoa II, Trưởng Đại hoc $Y$ Ḣ̀ Nô̂i, 94.

5. Nguyến Nhược Kim và Trân Quang Đạt (2008). Châm cứu và các phương pháp chửa bênh không dùng thuốc. Nhà xuất bản $Y$ hoc, Hà Nội, 37, 77, 114-115, 136-158,166-174, 223-225.

6. Nguyến Thị Ngọc Lan (2012). Bệnh học cơ xương khớp nội khoa. Nhà xuất bản Y học, Hà Nội, 138-151.

7. Childress M.A. và Becker B.A. Nonoperative Management of Cervical Radiculopathy. Am Fam Physician. 2016;93(9):746-754.

\title{
SO SÁNH CHỈ SỐ CORNELLVÀ SOKOLOW - LYON TRÊN ĐIỀN TÂM ĐỒ TRONG CHẨN ĐOÁN PHÌ ĐẠI THẤT TRÁI Ở BÊNH NHÂN TĂNG HUYẾT ÁP TIÊN PHÁT
}

\section{TÓM TẮT}

Mục tiêu: Khảo sát giá trị chẩn đoán phì đại thất trái (PĐTT) của chỉ số Cornell và Sokolow- Lyon trên điện tâm đồ (ĐTĐ) ở bệnh nhân tăng huyết áp (THA). Phương pháp nghiên cứu: Mổ tả cắt ngang. Đối

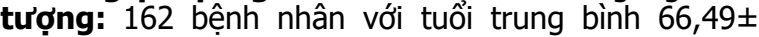
10,63 năm (nam giới chiếm 45,6\%) có THA được khảo sát PĐTT qua chỉ số Cornell và Sokolow-Lyontại Viện Tim Mạch Bach Mai thời gian từ 8/2020 đến 8/2021. Tiêu chuẩn phì đại thất trái được xác định qua siêu âm tim với khối lượng cơ thất trái $116 \mathrm{~g} / \mathrm{m}^{2}$ với nam và $96 \mathrm{~g} / \mathrm{m}^{2}$ với nữ. Kết quả: Diện tích của chỉ số điện thế Cornell so ba chỉ số điện thế Sokolow-Lyon, Sok P (Sokolow Lyon tích hợp) và Cornell $\mathrm{P}$ (Cornell tích hợp), khi chẩn đoán PĐTT có đối chiếu LVM/BSA: $(0,617$ so với 0,607 và $0,527,0,546$ ở nam; 0,555 so với 0,538 và $0,531,0,525$ ở nữ) với $p=N S$. Kết luận. Chỉ số điện thế Cornell có giá trị chẩn đoán PĐTT tương đương chỉ số Sokolow Lyon.

Tư khóa. Chỉ số Cornell và Sokolow Lyon, phì đại thất trái.

\section{SUMMARY}

\section{A COMPARISON OF CORNELL AND SOKOLOW - LYON ELECTROCARDIOGRAPHIC FOR LEFT VENTRICULAR HYPERTROPHY IN PATIENTS WITH HYPERTENSION}

Objectives. Survey the diagnostic value of left

\footnotetext{
${ }^{1}$ Bệnh viện Tỉnh Sơn La

²Bênh viện Tim Hà Nội

${ }^{3}$ Viện Tim mach ViêT Nam-Bênh viện Bạch Mai

Chịu trách nhiệm chính: Tạ Vắn Huyện

Email: hoanghuyenyk88@gmail.com

Ngày nhận bài: 6.8.2021

Ngày phản biên khoa hoc: 4.10 .2021

Ngày duyệt bài: 15.10.2021
}

ventricular hypertrophy of theCornell and SokolowLyon index on the electrocardiogram in hypertension patients. Methods. Cross-sectional descriptive study.

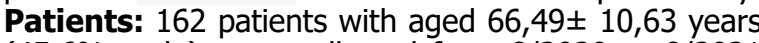
(45,6\% male) were collected from 8/2020 to 8/2021 at Vietnam National Heart Institute, the echocardiographic LVH which was defined as LVM index $\geq 116 \mathrm{~g} / \mathrm{m}^{2}$ in male patients and $\geq 96 \mathrm{~g} / \mathrm{m}^{2}$ in female patients. Results. The measured area under the curve of each area index of the Cornell voltage index is equivalent tothe three Sokolow-Lyon, Sok P (Sokolow Lyon Product) and Cornell P (Cornell Product) voltage indexes when diagnosing $\mathrm{LVH}$ with LVM/BSA comparison: 0.617 versus (vs) 0.607 and 0.527 vs 0.546 in male patients; 0.555 vs 0.538 and 0.531 vs 0.525 in female patientswith $p=N S$. Conclusions. The Cornell ECG criteria for the echocardiographic LVH had equivalent to SokolowLyon index in hypertension patients.

Key word. Cornell and Sokolow Lyon index, left ventricular hypertrophy.

\section{I. ĐẶT VẤN ĐỀ}

Phì đại thất trái (PĐTT) là một biến chứng của THA, cũng là yếu tố nguy cơ của các bệnh lý tim mạch khác. Nó làm tăng nguy cơ bệnh mạch vành lên 3 lần, suy tim lên hơn 13 lần, tai biến mạch máu não lên khoảng 6 lần và các nguy cơ động mạch ngoại biên, đột tử cũng 4-5 lần so với người không bị PĐTT. Để chẩn đoán PĐTT trên ĐTĐ có rất nhiêu chỉ số điện thế: Cornell, Sokolow Lyon, Norman,... nhìn chung chỉ đạt được độ đặc hiệu cao, còn độ nhạy thì lại rất thấp [1-3]. Đã có nhiêu nghiên cứu trên thế giới và Việt Nam nói về giá trị chẩn đoán PĐTT của các tiêu chí trên ĐTĐ nhưng so sánh riêng giữa tiêu chí điện thế Cornell và Sokolow Lyon thì 
chưa có nhiều. Chúng tôi thực hiện nghiên cứu này nhằm khẳng định giá trị của chỉ số điện thếCornell và Sokolow Lyon trong chẩn đoán PĐTT khi áp dụng trên bệnh nhân THA ở Việt Nam.

\section{II. ĐỐI TƯợNG VÀ PHƯƠ'NG PHÁP NGHIÊN CỨU}

Đối tượng nghiên cứu: Các bệnh nhân THA tới khám và điều trị tại Viện Tim mạch Việt Nam trong thời gian từ tháng 8/2020 đến 8/2021.

Tiêu chuẩn chọn bệnh nhân: Các bệnh nhân đến khám và điều trị tại viện Tim mạch ,được chẩn đoán THA theo tiêu chuẩn 2018 [4], được làm đồng thời cả SAT và ĐTĐ. Các bệnh nhẩn này đồng ý tham gia nghiên cứu.

Tiêu chuẩn loại trừ: $\mathrm{BN}$ tăng huyết áp thứ phát. Bệnh van tim: hẹp van tim nhe đến nặng, hở van vừa và nặng. Rối loạn nhịp tim, những trường hợp không phải nhịp xoang. Đái tháo đường.

Thiết kế nghiên cứu: Cắt ngang, mô tả.

Phương pháp chọn mẫu: Thuận tiên,tất cả bênh nhân thỏa mãn những tiêu chuẩn chọn mẫu sẽ đưa vào nghiên cứu theo trình tự thời gian.

Thu thập số liệu: Những biến số về tuổi, giới, tiền sử bệnh thu thập qua bệnh án. Chiều cao, cân nặng được đo trực tiếp bằng thước và cân bàn. Bệnh nhân được đo huyết áp được chẩn đoán THA sau đó sẽ் làm ĐTĐ: bằng máy đo 3 cần hiệu Schilercardiovit AI1, vận tốc máy đo là $25 \mathrm{~mm} / \mathrm{s}$, điện thế chuẩn là $10 \mathrm{~mm}$ bằng 1 milivolt. Bệnh nhân được nằm nghỉ 15 phút trước khi đo. Các chỉ số ĐTĐ khảo sát gồm: Sokolow-
Lyon: SV1 + RV5 hoặc RV6 $\geq 35 \mathrm{~mm}$, Cornell: $\mathrm{RaVL}+\mathrm{SV} 3 \geq 28 \mathrm{~mm}$ với nam ( $\geq 20 \mathrm{~mm}$ với nữ), Cornell tích hợp > $2440 \mathrm{~mm} * \mathrm{~ms}$, Sokolow Lyon tích hợp > 3340 mm*ms. Siêu âm tim: Đánh giá qua 5 mặt cắt lấy trung bình cộng của 5 mặt để tính khối lượng cơ thất trái được theo công thức Devereux (LVM = 1,04 x [ $\{$ LVDd + độ dày thành sau (LWd) + độ dày vách liên thất (IVSd) $\}^{3}$ LVIDd $\left.^{3}\right] \times 0,8+0,6$. Từ đó tính $L V M / B S A$ và LVM/chiều $\mathrm{caO}^{2,7}$. Ngưỡng phì đại: LVM/chiêu $\mathrm{cao}^{2,7} \geq 49 \mathrm{~g} / \mathrm{m}^{2,7}$ với nam, $\geq 45 \mathrm{~g} / \mathrm{m}^{2,7}$ với nữ). (LVMI: Khối lượng thất trái hiệu chỉnh theo chiều cao mũ $2,7\left(\mathrm{~g} / \mathrm{m}^{2,7}\right)$, theo Durex LVM/BSA $\geq$ $116 \mathrm{~g} / \mathrm{m}^{2}$ với nam và $\geq 96 \mathrm{~g} / \mathrm{m}^{2}$ với nữ $[5]$.

Xử lý thống kê. Sử dụng phần mềm SPSS 20.0. Tương quan được đánh giá bằng hệ số Pearson (R). Tính các giá trị của chỉ số ĐTĐ gồm: độ nhạy, độ đặc hiệu, giá trị tiên đoán dương, giá trị tiên đoán âm và độ chính xác. So sánh chỉ số Cornell với các chỉ số cùng khảo sát. So sánh các độ nhạy ở cùng một độ đặc hiệu $90 \%, 95 \%$ và $100 \%$. So sánh các đường cong ROC qua các diện tích nằm dưới các đường cong này.

\section{KẾT QUẢ NGHIÊN CứU}

Từ tháng 8/2020 đến 8/2021 chúng tôi thu thập được 162 bệnh nhân gồm 78 nam và 84 nữ, tuổi: cao nhất là 90 tuổi, thấp nhất là 27 tuổi, trung bình là $66,49 \pm 10,63$ tuổi. Giới: nam chiếm $48 \%$ và nữ chiếm $52 \%$.

Bảng 1. Đặc điểm nhân trắc học

\begin{tabular}{|c|c|c|c|c|}
\hline & Nam (n = 78) & Nữ (n = 84) & Tống (n=162) & p \\
\hline Tuối (năm) & $64,81 \pm 12,30$ & $68,05 \pm 8,59$ & $66,49 \pm 10,63$ & 0,052 \\
\hline Chiêu cao (cm) & $164,46 \pm 6,30$ & $154,06 \pm 5,12$ & $159,07 \pm 7,73$ & 0,000 \\
\hline Cân nặng (kg) & $62,08 \pm 9,83$ & $53,43 \pm 7,82$ & $57,59 \pm 9,82$ & 0,000 \\
\hline BMI (kg/m²) & $22.89 \pm 2,91$ & $22,48 \pm 2,88$ & $22,68 \pm 2,9$ & 0,364 \\
\hline Nhẹ cân (n,\%) & $8(10,3)$ & $6(7,1)$ & $14(8,6)$ & \multirow{2}{*}{0,828} \\
\hline Bình thường (n\%) & $35(44,9)$ & $43(51,2)$ & $78(48,1)$ & \\
\hline Thửa cân (n\%) & $30(38,5)$ & $30(35,7)$ & $60(37,0)$ & \\
\hline Béo phì (\%) & $5(6,4)$ & $5(6,0)$ & $10(6,2)$ & \\
\hline
\end{tabular}

Bảng 2: Tỷ lệ phi đại thất trái trên ĐTĐ:

\begin{tabular}{|c|c|c|c|}
\hline & Nam & Nữ & Chung \\
\hline Cor V & $3,8 \%$ & $6,0 \%$ & $4,9 \%$ \\
\hline Cor P & $3,81 \%$ & $1,2 \%$ & $2,5 \%$ \\
\hline Sok V & $10,1 \%$ & $8,3 \%$ & $9,2 \%$ \\
\hline Sok P & $5,1 \%$ & $2,4 \%$ & $3,7 \%$ \\
\hline
\end{tabular}

Bảng 3. Mối tương quan giữa bốn tiêu chí điện tâm đồ và hai loại chỉ số khối tâm thất trái ở nam giới

\begin{tabular}{|c|c|c|c|c|}
\hline \multirow{2}{*}{} & \multicolumn{2}{|c|}{ LVMI/BSA } & \multicolumn{2}{c|}{ LVMI/chî̂u cao $^{\mathbf{2 , 7}}$} \\
\cline { 2 - 5 } & $\mathbf{r}$ & $\mathbf{p}$ & $\mathbf{r}$ & $\mathbf{p}$ \\
\hline Sokolow-Lyon(mv) & 0,1758 & 0,1238 & 0,1892 & 0,0971 \\
\hline Sokolow-Lyon tích hợp (mm.ms) & 0,1166 & 0,3094 & 0,1229 & 0,2837 \\
\hline Cornell(mm) & 0,1973 & 0,0233 & 0,2130 & 0,0211 \\
\hline Cornell tích hợp(mm.ms) & 0,1450 & 0,2054 & 0,1535 & 0,1796 \\
\hline
\end{tabular}




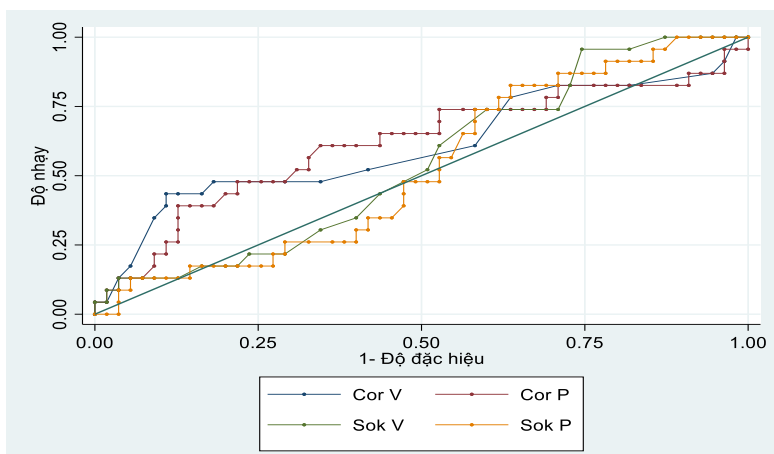

LVM/BSA

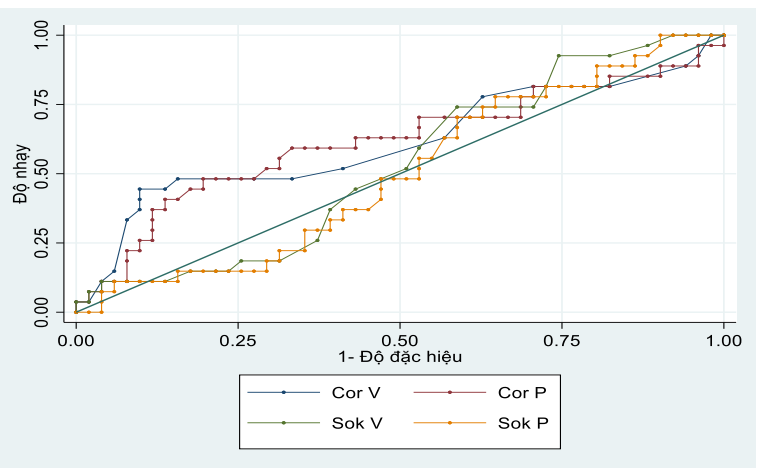

LVM/chiều cao ${ }^{2,7}$

Biểu đồ 1. Đồ thị đường cong ROC ở giới nam

\begin{tabular}{|c|c|c|c|c|}
\hline & \multicolumn{4}{|c|}{ P so sánh bắt că̆p giữa các AUC } \\
\hline & Sok V & Cor V & Sok P & Cor P \\
\hline Sok V & - & - & - & - \\
\hline Cor V & 0,2542 & - & - & - \\
\hline Sok P & 0,4595 & 0,1296 & - & - \\
\hline Cor P & 0,2664 & 0,9353 & 0,0915 & - \\
\hline
\end{tabular}

Nhận xét: sự khác biệt về giá trị chấn đoán giữa các chỉ số điện tâm đồ là chưa có ý nghĩa thống kê (tất cả p so sánh đều > 0,05)

Bảng 4. Mối tương quan giữa bốn tiêu chí điện tâm đồ và hai loại chỉ số khối tâm thất trái ở nữ giới

\begin{tabular}{|c|c|c|c|c|}
\hline & \multicolumn{2}{|c|}{ LVMI/BSA } & \multicolumn{2}{|c|}{ LVMI/chiêuu $\mathrm{CaO}^{2,7}$} \\
\hline & $\mathbf{r}$ & $\mathbf{p}$ & $\mathbf{r}$ & p \\
\hline Sokolow-Lyon(mv) & 0,0422 & 0,7030 & 0,0515 & 0,6415 \\
\hline Sokolow-Lyon tích hợp (mm.ms) & 0,0003 & 0,9982 & 0,0213 & 0,8576 \\
\hline Cornell $(\mathrm{mv})$ & 0,1352 & 0,0220 & 0,1417 & 0,0190 \\
\hline Cornell tích hợp(mm.ms) & 0,0817 & 0,4599 & 0,0766 & 0,4886 \\
\hline
\end{tabular}

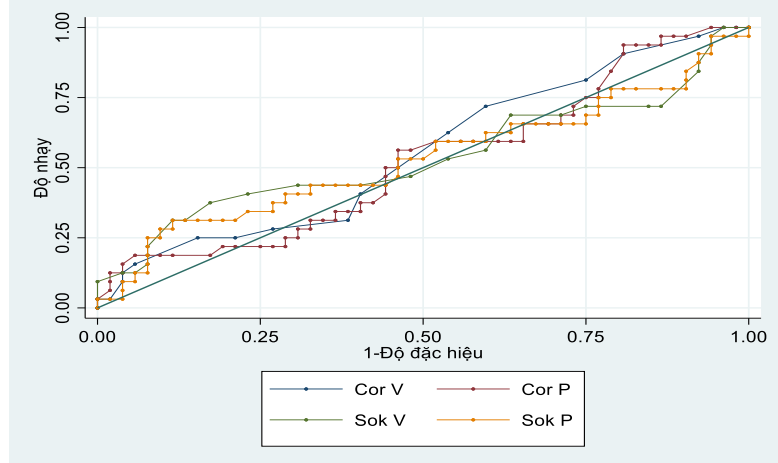

LVM/BSA

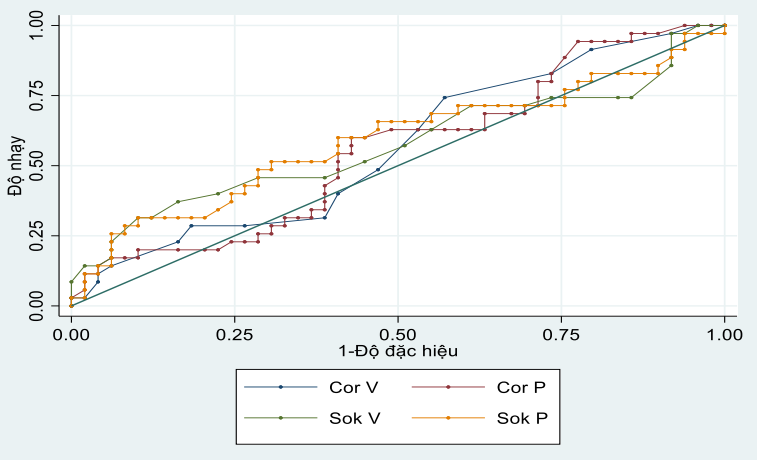

LVM/chiều $\mathrm{CaO}^{2,7}$

Biểu đồ 2. Đồ thị đường cong ROC ở giới nữ

\begin{tabular}{|c|c|c|c|c|}
\hline & \multicolumn{4}{|c|}{ P so sánh băt cặp giữa các AUC } \\
\hline & Sok V & Cor V & Sok P & Cor P \\
\hline Sok V & - & - & - & - \\
\hline Cor V & 0,8879 & - & - & - \\
\hline Sok P & 0,5162 & 0,7103 & - & - \\
\hline Cor P & 0,8136 & 0,8377 & 0,5834 & - \\
\hline
\end{tabular}

Nhận xét: sự khác biệt về giá trị chẩn đoán giữa các chỉ số điện tâm đồ là không có ý nghĩa thống kê $(p>0,05)$

\section{BÀN LUÂ̂N}

Phân bố tỉ lệ PĐTT theo giới: so sánh tỉ lệ PĐTT giữa hai giới có sự khác biệt không có ý nghĩa thống kê p>0,005

Tương quan giữa ĐTĐ và khối cơ thất trái trên siêu âm tim: Biểu đồ phân tán các phần tử tạo đám mây của 4 chỉ số đều có xu hướng là khối thất trái tăng thì các trị số ĐTÐ cũng tăng. Chúng tập trung có dạng đường 
thẳng tuyến tính thuận chiều. Biểu đồ đám mây của chỉ số Sokolow Lyon phân tán rộng nhất, cho giá trị hệ số tương quan thấp nhất( $R=0,17)$, còn biểu đồ đám mây của chỉ số Cornell thì tập trung quanh đường hồi quy, nên giá trị hể số tương quan cao hơn $(R=0.19)$ có ý nghĩa thống kê.

Độ nhay, độ đặc hiệu của 4 tiêu chí ĐTÐ: Chúng tôi thấy độ nhạy của 4 chỉ số ĐTĐ không khác nhau nhiêu và đều ở mức thấp, nhưng độ đặc hiệu của cả 4 tiêu chí ĐTĐ lại rất cao. Nhưng khi so sánh giá trị các chỉ số ĐTĐ ở cùng độ đặc hiệu: mặc dù độ nhạy của 4 chỉ số ĐTĐ là thấp nhưng khi so sánh chúng với nhau ở cùng độ đặc hiệu $90 \%, 95 \%$, và $100 \%$ thì chỉ số Cor V lại cho kểt quả cao hơn các chỉ số còn lại nhưng không có ý nghĩa thống kê.

So sánh đồ thị đường cong ROC của các chỉ số ĐTĐ: Chúng tôi thấy đường cong của chỉ số Cor $V$ nằm cao nhất ở cả 2 giới $(0,617$ ở nam; 0,525 ở nữ), đường cong của chỉ số Sok $V$ là thấp nhất, đặc biệt là ở giới nữ, mặc dù diện tích dưới đường cong của 4 tiêu chí ĐTÐ có khác nhau nhưng chưa có ý nghĩa thống kê và mức độ tương quan của từng chỉ số khi test chẩn đoán PĐTT lại ở mức yếu. Sau khi viết phương trình hồi quy cho các yếu tố ảnh hưởng đến chẩn đoán PĐTT chúng tôi thấy rằng: thời gian THA, chỉ số khối cơ thể (BMI), và có rối loạn lipid máu ảnh hưởng tới các chỉ số ĐTĐ, cụ thể là khi các yếu tố này tăng lên thì mức độ PĐTT cũng sẽ tăng lên.

\section{KẾT LUÂN}

Chỉ số điện thế Cornell có giá trị chẩn đoán PĐTT tương đương chỉ số Sokolow Lyon.

TÀI LIỆU THAM KHẢO
1. Sundström, J. et al. Echocardiographic and electrocardiographic diagnoses of left ventricular hypertrophy predict mortality independently of each other in a population of elderly men. Circulation 103, 2346-2351 (2001).

2. Molloy, T. J., Okin, P. M., Devereux, R. B. \& Kligfield, P. Electrocardiographic detection of left ventricular hypertrophy by the simple QRS voltageduration product. J. Am. Coll. Cardiol.20, 11801186 (1992).

3. Electrocardiographic detection of left ventricular hypertrophy using echocardiographic determination of left ventricular mass as the reference standard. Comparison of standard criteria, computer diagnosis and physician interpretation - PubMed. https:// pubmed.ncbi.nlm.nih.gov/6228571/.

4. Huỳnh Văn Minh, Phạm Gia Khải, Đặng Vặn Phước, Nguyến Lân Viête. Khuyến cáo về chẩn đoán và điêu trị THA 2018. Hội Tim mach hoc Viêt nam

5. Su, F.-Y. et al. A comparison of Cornell and Sokolow-Lyon electrocardiographic criteria for left ventricular hypertrophy in a military male population in Taiwan: the Cardiorespiratory fitness and HospItalization Events in armed Forces study. Cardiovasc. Diagn. Ther.7, 244-251 (2017).

6. Jj, N., Ta, A., Uf, N., C, N. \& Am, J. A metaanalytic evaluation of the diagnostic accuracy of the electrocardiographic Peguero-Lo Presti criterion for left ventricular hypertrophy. J. Clin. Hypertens. Greenwich Conn22, (2020).

7. Performance of Electrocardiographic Criteria for Echocardiographically Diagnosed Left Ventricular Hypertrophy in Chinese Hypertensive Patients - PubMed. https:// pubmed.ncbi.nlm.nih.gov/ 32484222/.

8. Sokolow, M. \& Lyon, T. P. The ventricular complex in left ventricular hypertrophy as obtained by unipolar precordial and limb leads. Am. Heart J.37, 161-186 (1949).

9. Giá trị của chỉ số norman trong chẩn đoán phì đại thất trái. https:// 123docz.net/ document/5950328-gia-tri-cua-chi-so-normantrong-chan-doan-phi-dai-that-trai.htm.

\title{
KẾT QUẢ ĐIỀU TRI HộI CHỨNG CHÈN ÉP KHOANG DƯỚI MỎM CÙNG VAI BẰNG TIÊM CORTICOSTEROID DƯớI HƯỚNG DẪN SIÊU ÂM
}

\author{
Hà Thanh Tâm ${ }^{1,2}$, Nguyễn Thị Ngọc Lan ${ }^{1}$, Phạm Hoài Thu ${ }^{1}$
}

\section{TÓM TẮT}

Muc tiêu nghiên cứu: Đánh giá kết quả điều trị hội chứng chèn khoang dưới mỏm cùng vai bằng tiêm

\footnotetext{
${ }^{1}$ Trường Đại học Y Hà Nội

²Bênh viện Trung ương Thái Nguyên

Chịu trách nhiệm chính: Phạm Hoài Thu

Email: phamhoaithu@hmu.edu.vn

Ngày nhận bài: 5.8.2021

Ngày phản biên khoa hoc: 4.10 .2021

Ngày duyệt bài: 15.10 .2021
}

corticosteroid dưới hướng dẫn của siêu âm và nhân xét một số tác dụng không mong muốn của liệu pháp.Đối tượng và phương pháp nghiên cứu: Nghiên cứu can thiêp theo dõi doc 29 bênh nhân với 34 khớp vai tổn thương, được chẩn đoán hội chứng chèn ép khoang dưới mỏm cùng vai và được tiêm 1 mũi Methylprednisolon acetat $40 \mathrm{mg}$ (Depomedrol 40 $\mathrm{mg}$ ) vào bao thanh dịch dưới mỏm cùng vai dướ hướng dẫn của siêu âm. Kết quả nghiên cứu: Sau 4 tuần điều trị có sự cải thiên về thang điểm VAS, EFA và góc dạng khớp vai. Đánh giá tại thời điểm trước tiêm và sau tiêm 4 tuần, VAS trung bình giảm từ 6,67 